\title{
Author Correction: Atom-by-atom fabrication with electron beams
}

Ondrej Dyck (D, Maxim Ziatdinov, David B. Lingerfelt (D), Raymond R. Unocic (D), Bethany M. Hudak (D), Andrew R. Lupini, Stephen Jesse and Sergei V. Kalinin (D)

Nature Reviews Materials (2019) https://doi.org/10.1038/s41578-019-0118-z Published online 07 June 2019

This article was originally published with an error in the acknowledgements section. The original text, "This work was supported by the Laboratory Directed Research and Development program of Oak Ridge National Laboratory, managed by UT-Battelle, LLC for the U.S. Department of Energy (O.D., M.Z., S.J.), Oak Ridge National Laboratory's Center for Nanophase Materials Sciences (CNMS), a U.S. Department of Energy Office of Science user facility (D.L. R.R.U., S.V.K.) and the U.S. Department of Energy, Office of Science, Basic Energy Sciences, Division of Materials Science and Engineering (B.M.H., A.R.L.).”, should have read: “This work was supported by the U.S. Department of Energy, Office of Science, Basic Energy Sciences, Materials Science and Engineering Division (B.M.H., A.R.L. S.V.K.), the Laboratory Directed Research and Development program of Oak Ridge National Laboratory, managed by UT-Battelle, LLC for the U.S. Department of Energy (O.D., M.Z., S.J.), and Oak Ridge National Laboratory's Center for Nanophase Materials Sciences (CNMS), a U.S. Department of Energy Office of Science user facility (D.L. R.R.U.)." This has been corrected in the online version and in the pdf.

https://doi.org/10.1038/s41578-020-0188-y I Published online 20 February 2020

(๑) Springer Nature Limited 2020 